\title{
CALCIFICAÇÕES ORBITÁRIAS INCIDENTAIS NA TOMOGRAFIA COMPUTADORIZADA*
}

\author{
Dalton Yukio A. Fugita ${ }^{1}$, Daniela Nogueira Cruz ${ }^{1}$, Alessandro Cappucci ${ }^{2}$, Márcia Mayumi \\ Arakava ', Maria Carolina Guimarães ', Paulo Góes Manso ${ }^{3}$, Ângela Maria B. Wolosker ${ }^{4}$, \\ Hélio Kiitiro Yamashita ${ }^{5}$
}

Resumo Estudamos, retrospectivamente, tomografias computadorizadas de órbita de 75 pacientes, de maneira a identificar a presença de calcificações incidentais (esclerais e do aparato troclear). Estes achados devem ser incluídos no vasto diagnóstico diferencial das calcificações orbitárias. A familiaridade com seus achados de imagem pode ajudar o radiologista a diferenciá-las do diagnóstico de corpo estranho orbitário.

Unitermos: Órbita. Tomografia computadorizada. Calcificação.

\begin{abstract}
Incidental orbital calcifications on computed tomography scans.
We retrospectively studied the computed tomography scans of the orbit in $\mathbf{7 5}$ patients in order to identify the presence of incidental calcifications (scleral and trochlear apparatus calcifications). These imaging findings should integrate the vast list of differential diagnosis of orbital calcifications, as they may help radiologists to distinguish these calcifications from orbital foreign bodies.

Key words: Orbit. Computed tomography. Calcification.
\end{abstract}

\section{INTRODUÇÃO}

As calcificações orbitárias apresentam um extenso diagnóstico diferencial, que inclui neoplasias, doenças metabólicas, trauma, entre outras condições. Porém, em alguns casos, tais calcificações podem ser apenas um achado radiológico, não estando necessariamente relacionadas à doença de base ou, então, não provocando quadro clínico específico. Este tipo de calcificação pode ser classificado como incidental e, como exemplo, são citadas as calcificações esclerais e as do aparato troclear do músculo oblíquo superior ${ }^{(1-3)}$.

\section{MATERIAIS E MÉTODOS}

Foram estudadas, retrospectivamente, as tomografias computadorizadas de órbita de 75 pacientes atendidos no Hos-

\footnotetext{
* Trabalho realizado no Departamento de Diagnóstico por Imagem (DDI) da Universidade Federal de São Paulo/ Escola Paulista de Medicina (Unifesp/EPM), São Paulo, SP.

1. Médicos Pós-graduandos do DDI-Unifesp/EPM.

2. Médico Residente do DDI-Unifesp/EPM.

3. Médico do Setor de Órbita do Departamento de Oftalmologia da Unifesp/EPM.

4. Chefe do Setor de Radiologia de Cabeça e Pescoço do DDI-Unifesp/EPM.

5. Professor Doutor do DDI-Unifesp/EPM, Setor de Radiologia de Cabeça e Pescoço.

Endereço para correspondência: Dr. Dalton Yukio A. Fugita. Rua Napoleão de Barros, 800, Vila Clementino. São Paulo, SP, 04024-002.

Aceito para publicação em 24/7/2000.
}

pital São Paulo, no período de janeiro de 1998 a junho de 1999, a fim de determinar a presença de calcificações incidentais. Desse total, foram considerados para análise apenas os estudos de 55 pacientes adultos maiores de 20 anos, resultando num grupo cuja idade média era de 49,1 anos, sendo 39 pacientes do sexo masculino $(70,9 \%)$ e 16 do sexo feminino $(29,1 \%)$

Desse total de 55 pacientes adultos, foram consideradas para estudo cerca de 103 órbitas, sendo que sete órbitas foram excluídas por estarem deformadas por condições diversas como trauma, tumores e enucleação.

As indicações para realização das 55 tomografias foram infecções $(23,63 \%)$, tumores $(18,1 \%)$, trauma $(20 \%)$, oftalmopatia endócrina $(14,5 \%)$, pseudotumor $(5,45 \%)$, iatrogenia $(1,8 \%)$ e indeterminadas/outras $(16,3 \%)$.

\section{RESULTADOS}

Detectamos a presença de seis calcificações incidentais em três pacientes (5,4\% do total de pacientes e $5,82 \%$ do total de órbitas estudadas). Destas seis calcificações, quatro eram calcificações esclerais, bilaterais e anteriores à inserção do músculo reto medial, e encontradas em duas pacientes do sexo feminino, de 80 e 91 anos de idade, o que cor- responde a $3,6 \%$ dos pacientes e a $3,88 \%$ das órbitas estudadas (Figura 1).

Em relação à calcificação do aparato troclear do músculo oblíquo superior, esta foi encontrada em apenas uma paciente do sexo feminino, de 42 anos de idade, sendo bilateral e representando $1,8 \%$ do total de pacientes e $1,94 \%$ das órbitas estudadas (Figura 2).

Nos pacientes com idade inferior a 20 anos, excluídos da análise, não foram detectadas calcificações incidentais.

\section{DISCUSSÃO}

As calcificações incidentais fazem parte do vasto diagnóstico diferencial das calcificações orbitárias e tornam-se importantes nos casos eventuais em que se necessita sua distinção com o diagnóstico de corpo estranho ${ }^{(1,4,5)}$. Nossos resultados estão de acordo com a escassa literatura, que refere uma relativamente baixa incidência deste tipo de calcificação, variando de $3 \%$ a $6,2 \%$ para as calcificações esclerais ${ }^{(1,2)}$ e $3 \%$ para as calcificações do aparato troclear ${ }^{(2)}$.

As causas deste tipo de calcificação ainda não estão totalmente determinadas, podendo estar relacionadas a condições degenerativas e ao próprio processo de envelhecimento ${ }^{(4)}$. Em relação às calcificações do aparato troclear, estas podem estar associadas ao diabetes 


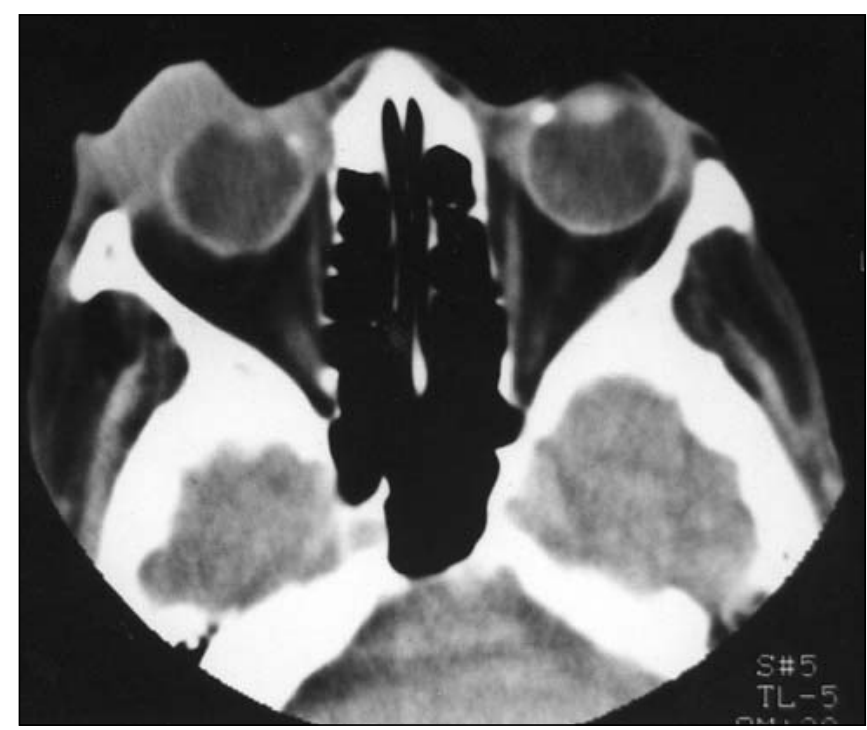

Figura 1. Calcificação escleral bilateral em paciente de 91 anos de idade com carcinoma espinocelular invadindo a órbita direita.

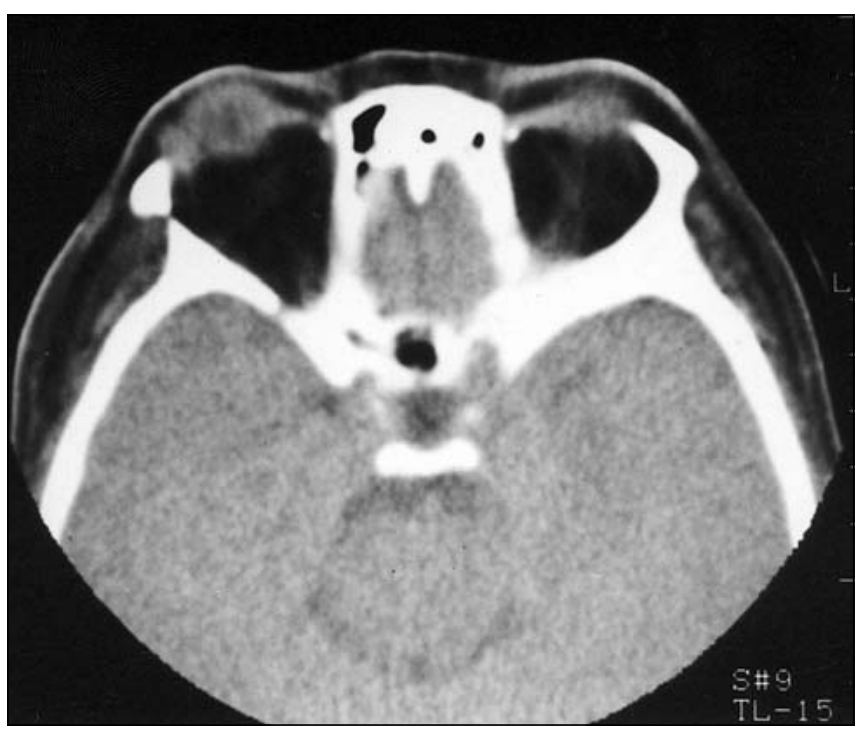

Figura 2. Calcificação bilateral do aparato troclear em paciente de 42 anos de idade com doença de Graves. quando presentes em indivíduos jovens, com idade inferior a $40 \operatorname{anos}^{(3)}$.

\section{CONCLUSÃO}

Devido aos poucos dados na literatura a respeito das calcificações incidentais, destacamos a importância de o radiologista estar ciente deste diagnós- tico diferencial, tendo em vista, particularmente, a sua distinção com corpo estranho orbitário ${ }^{(\mathbf{1})}$.

\section{REFERÊNCIAS}

1. Gordon RN, Slamovits TL, Rosenbaum PS, Bello J. Calcified scleral plaques imaged on orbital computed tomography. Am J Ophthalmol 1999; 127:461-3.

2. Murray JL, Hayman LA, Tang RA, Schiffman JS. Incidental asymptomatic orbital calcifications. J
Neuro-ophthalmol 1995;15:203-8.

3. Hart BL, Spar JA, Orrison WW Jr. Calcification of the trochlear apparatus of the orbit: $\mathrm{CT}$ appearance and association with diabetes and age. AJR 1992; 159:1291-4.

4. O'Steen WK, Brodish A. Scleral calcification and photoreceptor cell death during aging and exposure to chronic stress. Am J Anat 1990;189:62-8.

5. Caldemeyer KS, Smith RR, Edwards-Brown MK. Familial hypophosphatemic rickets causing ocular calcification and optic canal narrowing. AJNR 1995; 16:1252-4. 